Artículo de Revisión

Cómo citar: M. Castañeda, C. Camacho y J. Rativa. "Análisis de las variables que influyen en el desbordamiento del río Frío en el municipio de Campoalegre, Huila (Colombia)". Inventum, Vol 16, No. 30, pp. 31-39. doi: 10.26620/ uniminuto.inventum.16.30.2021.31-39

Editorial: Corporación Universitaria Minuto de Dios - UNIMINUTO.

ISSN: $1909-2520$

eISSN: 2590-8219

Fecha de recibido: Enero 10 de 2021 Fecha de aprobado: Febrero 10 de 2021 Fecha de publicación: Marzo 10 de 2021

Conflicto de intereses: los autores han declarado que no existen intereses en competencia.
Mónica Sofía Castañeda Espinosa u20152139583@usco.edu.co https://orcid.org/0000-0001-6399-7192 Universidad Surcolombiana (Neiva, Hulia), Programa de Matemática Aplicada. Grupo de investigación Henry Poincaré, semillero de Investigación.

Cindy Johanna Camacho Hernández u20151137282@usco.edu.co https://orcid.org/0000-0003-4587-1638. Universidad Surcolombiana (Neiva, Hulia), Programa de Matemática Aplicada. Grupo de investigación Henry Poincaré, semillero de Investigación.

Juan Felipe Rativa Ortega u20152143288@usco.edu.co https: / / orcid.org/0000-0002-5487-5609 Universidad Surcolombiana (Neiva, Hulia), Programa de Matemática Aplicada. Grupo de investigación Henry Poincaré, semillero de Investigación.

\section{Análisis de las variables que influyen en el desbordamiento del río Frío en el municipio de Campoalegre, Huila (Colombia)}

\author{
Analysis of the variables that influence \\ the overflow of the río Frío \\ in the municipality of Campoalegre, \\ Huila (Colombia)
}

\author{
Análise das variáveis que influenciam \\ o transbordamento do río Frío \\ no município de Campoalegre, \\ Huila (Colômbia)
}

\begin{abstract}
Resumen
En este estudio se realizó una abstracción teórica de los conceptos básicos sobre meteorología y la medición de cada una de sus variables, además, se analizó cada uno de los datos suministrados por el IDEAM, con el fin de determinar la variable con mayor incidencia en la creciente de la cuenca hídrica del río Frío, con ayuda del programa Weka. De los resultados de la simulación en el software experto en minería de datos, se puede concluir que el factor dominante es la precipitación, debido a que a mayor grado de precipitación es más alto el riesgo de que ocurra una inundación en el municipio de Campoalegre (Huila), y el resto de las variables afectarían directamente la precipitación. En esta dirección, este proyecto propone estudiar las causas del desbordamiento del río Frío en la región del municipio de Campoalegre, ocurrido el 22 de febrero de 2017 y que ocasionó daños materiales a la comunidad.
\end{abstract}

Palabras clave: cambio climático, conceptos meteorológicos, cuenca hídrica, río Frío, Weka.

\begin{abstract}
In this study, a theoretical abstraction of the basic concepts of meteorology and the measurement of each of its variables was carried out.In addition, each of the data provided by IDEAM was analyzed in order to determine the variable with the greatest incidence in the growing water basin of the Frío River, with the help of the Weka program. From the results of the simulation in the expert data mining software, it can be concluded that the dominant factor is precipitation, because the higher the degree of
\end{abstract}


precipitation, the higher the risk of a flood occurring in the municipality of Campoalegre (Huila), and the rest of the variables would directly affect precipitation. In this direction, this project proposes to study the causes of the overflow of the Frío River in the Campoalegre municipality region, which occurred on February 22, 2017 and caused material damage to the community.

Keywords: climate change, meteorological concepts, río Frío, water basin, Weka.

\section{Resumo}

Neste estudo, foi realizada uma abstração teórica dos conceitos básicos da meteorologia e a medição de cada uma de suas variáveis. Além disso, cada um dos dados fornecidos pelo IDEAM foi analisado a fim de determinar a variável com maior incidência no crescimento. bacia hidrográfica do rio Frío, com a ajuda do programa Weka. A partir dos resultados da simulação no software especialista em mineração de dados, pode-se concluir que o fator dominante é a precipitação, pois quanto maior o grau de precipitação, maior o risco de ocorrência de inundação no município de Campoalegre (Huila), e o resto das variáveis afetariam diretamente a precipitação. Nesse sentido, este projeto se propõe a estudar as causas do transbordamento do Rio Frío na região do município de Campoalegre, ocorrido em 22 de fevereiro de 2017 e que causou danos materiais à comunidade.

Palavras-chave: bacia hidrográfica, conceitos meteorológicos, mudanças climática, río Frío, Weka. 


\section{INTRODUCCIÓN}

El calentamiento global es una realidad que incide directamente en la calidad de vida, por tal motivo es oportuno determinar su causa y efecto, gracias a las matemáticas aplicadas en la naturaleza. Con la biomatemática se puede determinar el comportamiento en los ecosistemas y los factores que involucran su transformación con el paso del tiempo [1], [2].

Esta investigación es importante ya que es necesaria para analizar, determinar e interpretar el comportamiento de los ríos a lo largo del tiempo. Además, ayuda a precisar cuáles afluentes sufren cambios drásticos por la contaminación y las precipitaciones desde su nacimiento hasta su desembocadura, así como a conocer cuáles son los riesgos que existen para las personas que viven a sus alrededores y cómo influye y afecta el ecosistema en general [3]. De tal forma que, con la biomatemática se puede simular el recorrido de un río y sus resultados al sufrir cualquier tipo de cambio en su cuenca, para así concluir cuales son las mejores herramientas y acciones que se deben tomar para contrarrestar los riesgos de vida y subsistencia del hábitat natural [4].

El desbordamiento de los ríos ocurre cuando se excede la capacidad de los canales para conducir el agua y, por lo tanto, se desbordan las márgenes del río, causando severos problemas sociales, ambientales y económicos [5].

Asociado a lo anterior, se han registrado numerosos cambios en el clima a largo plazo. Estos cambios incluyen los posibles aumentos en la intensidad de los ciclones tropicales, en las ondas de calor y un aumento en la frecuencia de eventos extremos como sequías y lluvias torrenciales. Por ello se llevó a cabo el estudio minucioso realizado en la fuente hídrica rio Frio del municipio de Campoalegre (Huila).

\section{LOCALIZACIÓN DEL PROYECTO}

El municipio de Campoalegre está ubicado en el centro del departamento del Huila, con una distancia de $22 \mathrm{~km}$ entre este municipio y la ciudad de Neiva, medida tomada sobre la vía troncal del sur, que une la mayoría de los municipios regionales.

Tiene una extensión de $661 \mathrm{~km}^{2}$, según el Instituto Geográfico Agustín Codazzi (IGAC), el punto más bajo del municipio se encuentra en la confluencia del río Neiva con el río Magdalena y su altura es de 456 m s.n.m. La cota más alta corresponde al cerro Cresta de Gallo, ubicado en el ecosistema estratégico de la Siberia, a 3250 m s.n.m., que comparte con los municipios de Rivera y Algeciras.

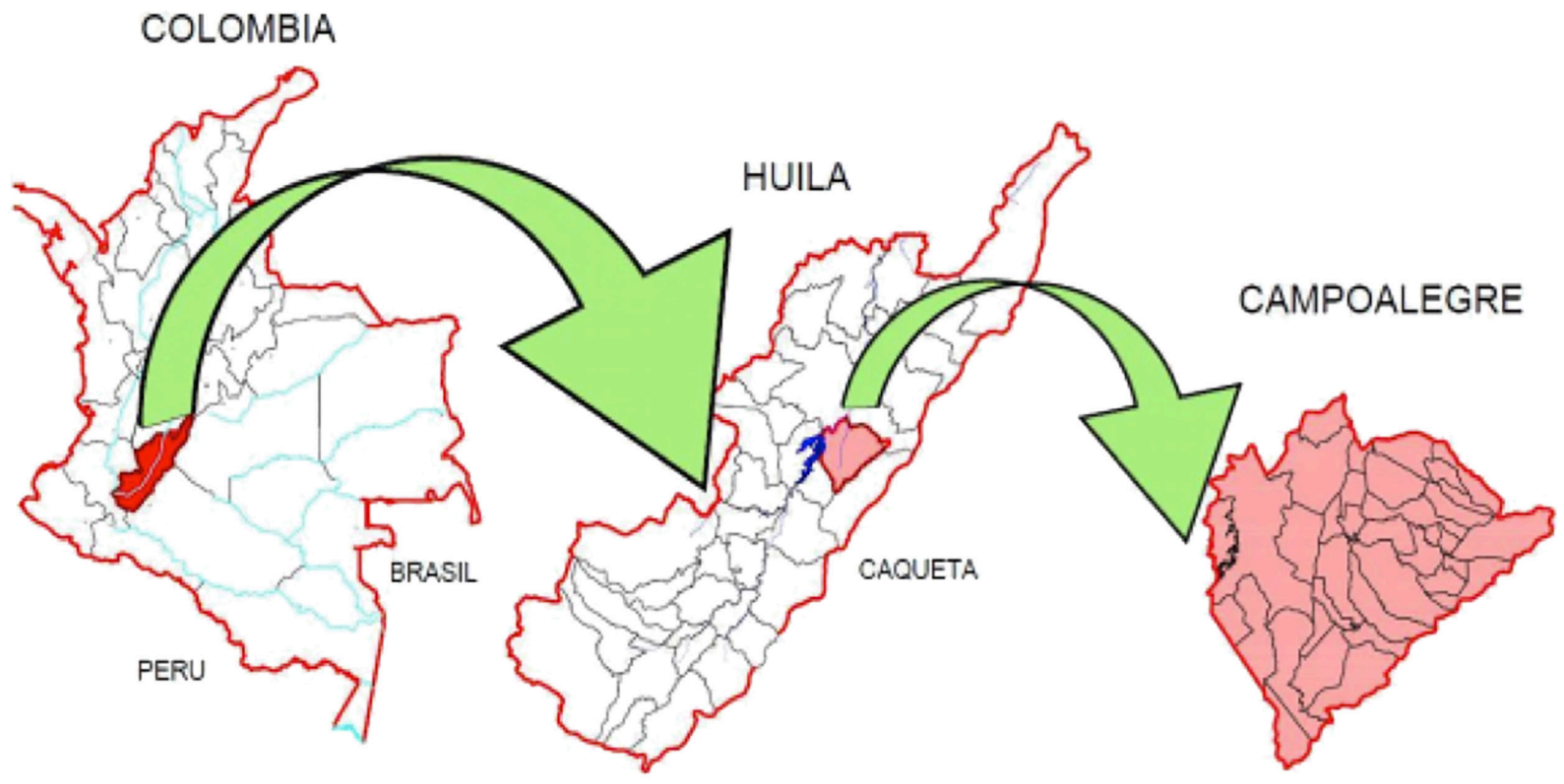

Figura 1. Localización geográfica de Campoalegre.

Fuente: [6]. 
La altura promedio de la ciudad es de 525 m s.n.m., y su temperatura media de $27{ }^{\circ} \mathrm{C}$ y con una precipitación media de 1254 milímetros.

El municipio de Campoalegre - Huila, limita al norte con el municipio de Rivera, al oriente con el municipio de Algeciras, al sur con el municipio de Hobo y al occidente con los municipios de Hobo y Yaguará. "Según las coordenadas geográficas del IGAC, el territorio del municipio de Campoalegre se inicia al sur a los $2^{\circ} 31^{\prime}$ y termina a los $2^{\circ} 47^{\prime}$ de latitud norte y en el este principia a los $75^{\circ} 12^{\prime}$ y termina a los $75^{\circ} 26^{\prime}$ de longitud oeste de Greenwich. La situación geográfica de su plaza principal corresponde a los $2^{\circ} 41^{\prime} 20^{\prime \prime}$ de latitud norte y a $75^{\circ}$ 14 ' $33^{\prime \prime}$ de longitud al occidente del meridiano de Santa fe de Bogotá". [15]

\section{MARCO REFERENCIAL}

Algunos conceptos y definiciones que tomamos para conocer el comportamiento atmosférico del tiempo y clima de la región de Campoalegre son los siguientes:

\section{A. Meteorología}

La meteorología como ciencia interdisciplinaria, es la encargada del estudio de la atmósfera y sus fenómenos, en particular sobre las condiciones del tiempo y del clima. [7]

\section{B. Clima}

Es el conjunto fluctuante de las condiciones atmosféricas, caracterizado por los estados y evoluciones del tiempo, en un periodo y región dados, y controlado por factores forzantes y determinantes, y por la interacción entre los diferentes componentes del sistema climático como lo son: la atmósfera, la hidrosfera, la litosfera, la criósfera, la biosfera y antropósfera) [7].

\section{Precipitación}

Se conoce como precipitación a la cantidad de agua que cae a la superficie terrestre y que proviene de la humedad atmosférica, en varios estados (estado líquido o sólido). Además, es uno de los procesos meteorológicos fundamentales para la Hidrología, y junto a la evaporización conforman la interacción de la atmosfera con el agua superficial en el ciclo hidrológico del agua. [17]

\begin{tabular}{c|l}
\hline Tipo & \multicolumn{1}{c}{ Definición } \\
\hline Llovizna & $\begin{array}{l}\text { Precipitación cuasi uniforme compuesta exclusivamente de pequeñas gotas de agua (diámetro } \\
\text { menor de } 0,5 \mathrm{~mm} \text { ) muy numerosas, que pueden reducir la visibilidad en mayor medida que la } \\
\text { lluvia ligera. }\end{array}$ \\
\hline Lluvia & $\begin{array}{l}\text { Precipitación de partículas de agua líquida en forma de gotas de diámetro mayor de 0,5 mm o } \\
\text { más pequeñas si caen de forma dispersa. }\end{array}$ \\
\hline Chubasco & $\begin{array}{l}\text { Lluvia formada por gotas de agua subfundida que se congela al impactar sobre un objeto. } \\
\text { Los chubascos se caracterizan por su comienzo y final repentinos, generalmente por grandes y } \\
\text { rápidos cambios de intensidad. Como sinónimo de chubasco puede usarse el término aguacero. }\end{array}$ \\
\hline Nieve & $\begin{array}{l}\text { Precipitación de cristales de hielo aislados o aglomerados. La cota de nieve es aquella a } \\
\text { partir de la cual la precipitación cae en forma de nieve, independientemente de si cuaja o no } \\
\text { a ese nivel. }\end{array}$ \\
\hline Granizo & $\begin{array}{l}\text { Precipitación de pequeños glóbulos o trozos de hielo (pedrisco) con diámetros de entre } 5 \\
\text { mm y } 50 \text { mm o algunas veces más, y que caen separados o agrupados irregularmente. }\end{array}$ \\
\hline
\end{tabular}

Tabla I. Tipos de precipitación. Fuente: adaptada de [8]. 


\section{Temperatura}

La temperatura es una magnitud física la cual determina una medida del grado de caliente o frio relativo, y que refiere, las variaciones de calor que presenta un cuerpo o un medio. [18]

En este sentido los términos que hay que utilizar son:

- "Pocos cambios o cambios ligeros": Variaciones de $\pm 2{ }^{\circ} \mathrm{C}$.

- “Aumento/descenso": Variaciones de entre $3{ }^{\circ} \mathrm{C}$ a $5{ }^{\circ} \mathrm{C}$.

- “Aumento/descenso notable": Variaciones de entre $6{ }^{\circ} \mathrm{C}$ a $10{ }^{\circ} \mathrm{C}$.

- "Aumento/descenso extraordinario": Variaciones superiores $\mathrm{a} \pm 10{ }^{\circ} \mathrm{C}$

Helada: Fenómeno que se produce cuando la temperatura del aire es igual o inferior a $0{ }^{\circ} \mathrm{C}$.

Las heladas se clasifican en:

- Débiles: cuando la temperatura esté entre $0{ }^{\circ} \mathrm{C}$ y $-4{ }^{\circ} \mathrm{C}$.

- Sin adjetivar: Cuando la temperatura esté entre -4 y $-8{ }^{\circ} \mathrm{C}$.

- Fuertes: Cuando la temperatura sea inferior a $-8{ }^{\circ} \mathrm{C}[8$, p. 28$]$.

\section{E. Humedad atmosférica}

La humedad atmosférica es la cantidad de vapor de agua disuelto en el aire del ambiente y depende de la temperatura. [19]

\section{F. Viento}

Es el aire del movimiento y se representa por un vector que puede ser descompuesto un un componente horizontal o vertical. El primero se conoce como viento, y el segundo, se le denomina corriente ascendente.

Para identificar el viento, es necesario determinar su dirección y velocidad. El primer concepto se encuentra asociado con la dirección del mismo y se define como aquella de donde procede y se expresa en grados sexagesimales contados en el sentido que giran las manecillas del reloj, a partir del norte geográfico, utilizando los rumbos de la rosa de los vientos. En este sentido, las direcciones principales son: Norte, Noreste, Este, Sureste, Sur, Suroeste, Oeste y Noroeste. Por otro lado, es oportuno referir que la velocidad del viento es la distancia que recorre una partícula de aire en la unidad de tiempo expresada en metros por segundo $(\mathrm{m} / \mathrm{s})$, kilómetros por hora $(\mathrm{km} / \mathrm{h})$ o nudos y que cuando es inferior a $0,5 \mathrm{~m} / \mathrm{s}$ se dice que el viento está en calma. [7]

\section{G. Brillo solar}

El brillo solar o insolación, se encuentra asociado con la cantidad de tiempo que es expuesta la superficie del suelo a través de la radiación solar directa. [7]

\section{H. Humedad relativa}

La humedad relativa se conoce como el equilibrio entre el agua real en el aire comparada con la cantidad de vapor de agua necesaria para la saturación a la temperatura correspondiente, y es considerada, como una medida que permite saber que tan húmedo o seco se encuentra el aire. [16]

\section{Tensión de vapor}

La tensión de vapor se define como la presión que tendría el vapor de agua si ocupara él solo el volumen ocupado por el aire húmedo. El valor en cantidad es limitado, y se dice que cuando lleva a ese nivel el aire es saturado. La presión de vapor así es la tensión de vapor de saturación (es) y depende únicamente de la temperatura.[9]

\section{J. Punto de rocío}

Conocido también como la temperatura de rocío, es la más alta temperatura a la que empieza a condensarse el vapor de agua contenido en el aire [16]

\section{K. Evaporación}

La evaporación es entendida como la máxima temperatura que alcanza un líquido para pasar al 
estado gaseoso. En este sentido, la circulación de los cuerpos de agua hacia la atmósfera genera a su vez procesos de saturación que conllevan a la probabilibdad de precipitaciones [10]

\section{Nubosidad}

La Nubosidad es la fracción de cielo cubierto de nubes de un cierto género, de una cierta especie, de una cierta variedad, de una cierta capa o de una combinación de nubes. En este sentido, el Cielo es el espacio atmosférico, ajeno a la corteza terrestre, que vemos desde nuestro lugar de observación, y que por lo tanto, hay múltiples cielos en una misma región por lo cual se habla de "cielos nubosos" o de alguna otra expresión que incluya la palabra cielo o cielos en un boletín de información meteorológica. [8]

\begin{tabular}{|c|c|}
\hline Tipo & Definición \\
\hline Nubes altas & $\begin{array}{l}\text { Precipitación cuasi uniforme compuesta exclusivamente de pequeñas gotas de agua } \\
\text { (diámetro menor de } 0,5 \mathrm{~mm} \text { ) muy numerosas, que pueden reducir la visibilidad en } \\
\text { mayor medida que la lluvia ligera. }\end{array}$ \\
\hline Nubes medias y altas & $\begin{array}{l}\text { Pueden producir precipitaciones y, en el caso de que tengan poco espesor, pueden } \\
\text { dejar pasar los rayos del sol. }\end{array}$ \\
\hline Nubes bajas & $\begin{array}{l}\text { En general serán estratos o estratocúmulos. Un caso especial serían las nieblas y las } \\
\text { brumas, aunque estos fenómenos serán tratados en el apartado de visibilidad. }\end{array}$ \\
\hline Nubosidad de evolución (diurna) & $\begin{array}{l}\text { Aquella que se desarrolla en las horas centrales del día por efecto del calentamiento } \\
\text { del suelo y que desaparece por la noche. }\end{array}$ \\
\hline Nubosidad de estancamiento (retención) & $\begin{array}{l}\text { Nubes bajas que persisten largo tiempo en un mismo lugar, generalmente en la costa o } \\
\text { en la montaña, debido principalmente a la orografía. }\end{array}$ \\
\hline
\end{tabular}

Tabla 2. Tipos de nubosidad.

Fuente: adaptado de [8].

\section{METODOLOGÍA}

\section{Tipo de estudio}

El presente estudio es una investigación descriptiva con enfoque cualitativo y cuantitativo y está delimitado por la problemática del desbordamiento sobre la cuenca hídrica río Frío en la zona de Campoalegre, Huila [11], [12].

\section{Técnica de estudio}

Se hace una recolección de datos de las variables que influyen en el desbordamiento de la cuenca hídrica, datos suministrados por las estaciones meteorológicas las cuales registran los parámetros climáticos del sitio donde están instaladas, ellas son: Hacienda Potosí, Los Rosales e Hidrobetania. Dichas variables fueron polucionadas en forma numérica y se hizo el respectivo cambio a variables cualitativas, luego fueron digitadas en Excel, para posteriormente ser utilizadas en el programa Weka y hacer un análisis mediante un árbol de decisión con base en los datos suministrada por el IDEAM.

Después, se realizó una abstracción teórica de los conceptos básicos sobre meteorología y la medición de cada una de sus variables [13], [14].

Una vez hecho el análisis de los datos, se procedió a identificar las variables con mayor incidencia en la creciente de la cuenca hídrica río Frío y, por ende, en el cambio climático del mismo con la ayuda del programa matemático Weka [5].

\section{RESULTADOS}

A continuación, se presenta la aplicación que se utilizó en el programa Weka [13] y los resultados que arrojó: 
- EXPLORE: el modo explorador es el modo más usado y descriptivo. Este permite realizar operaciones sobre un solo archivo de datos.

El explorador permite tareas de:

1. Preprocesador de los datos y aplicación de filtros.

2. Clasificación.

\section{Clustering.}

4. Búsqueda de asociaciones.

5. Selección de atributos.

6. Visualización de datos.

Después se ingresó la base de datos (anexo) al programa Weka para generar el árbol de decisión en J48.

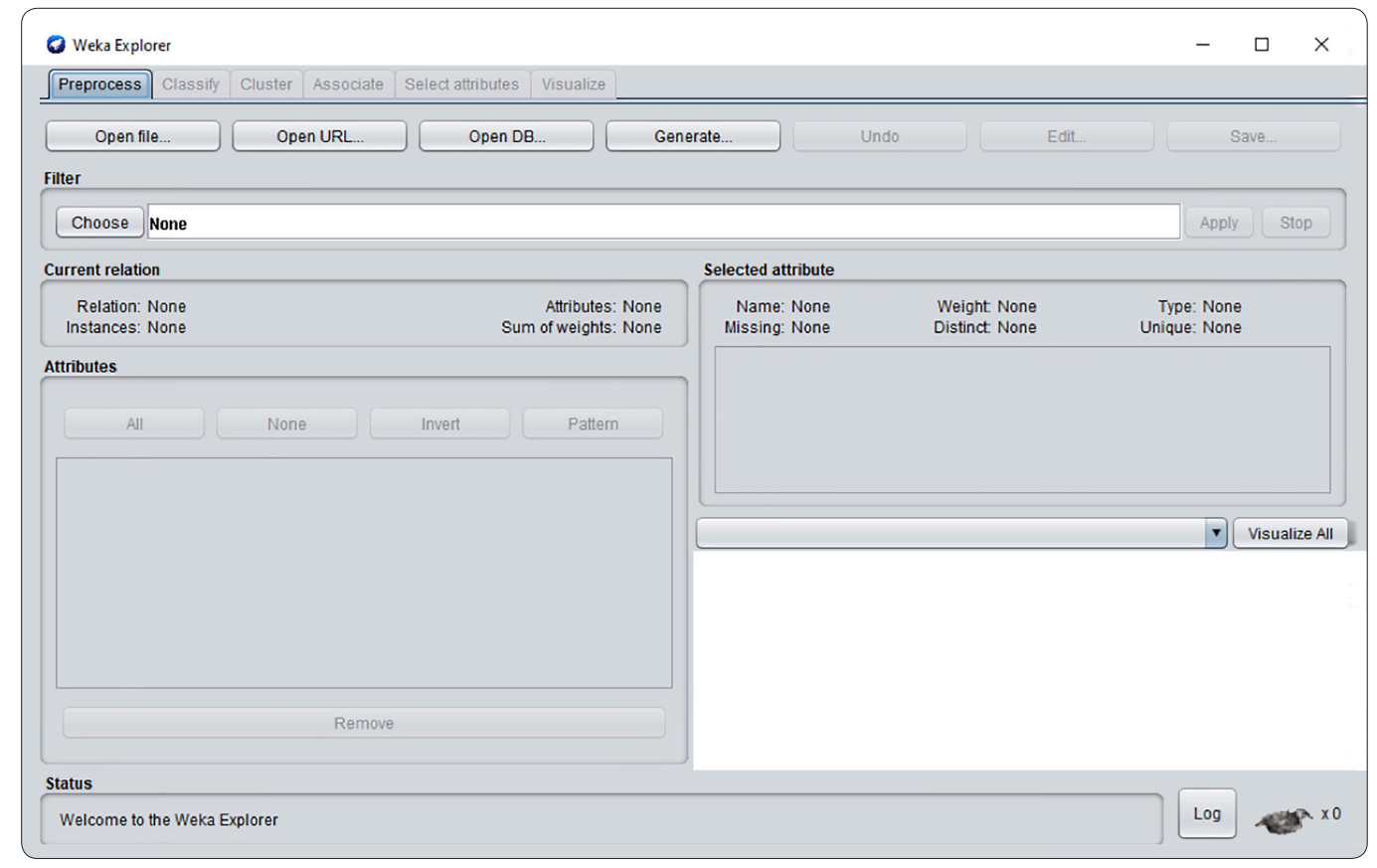

Figura 2. Escritorio de Weka con el modo explore.

Fuente: elaboración propia.

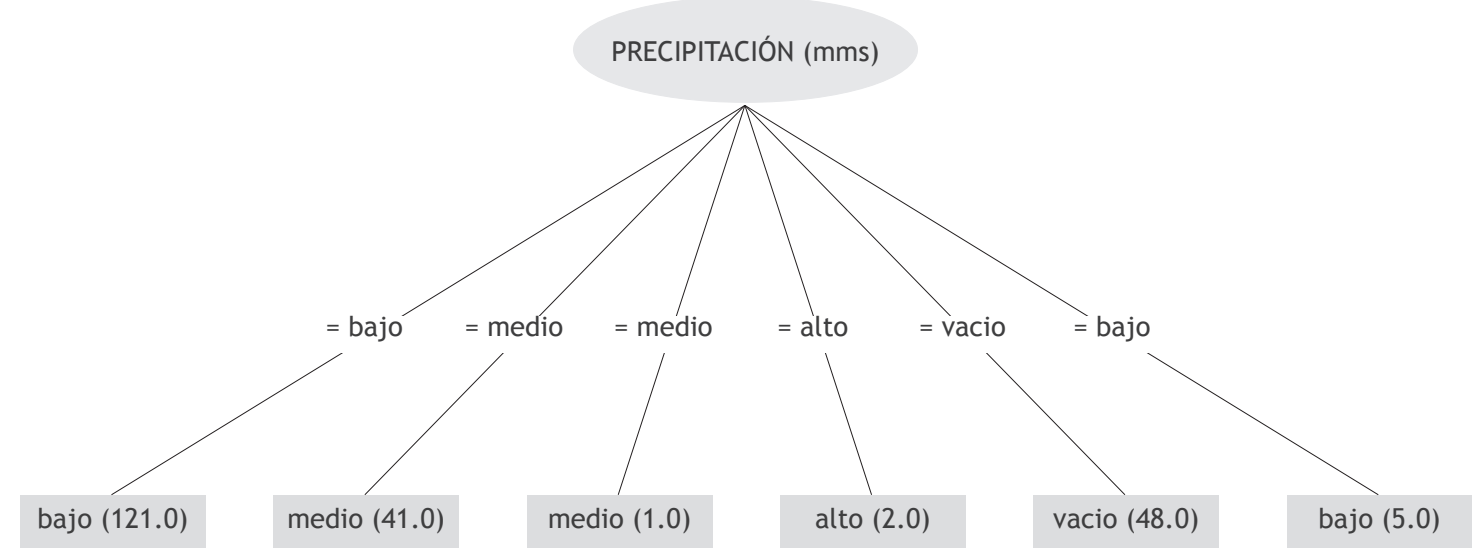

Figura 3. Árbol de decisión.

Fuente: elaboración propia. 


\section{A. Primeros resultados}

- El factor dominante es la precipitación debido a que cuando es alta el grado de que ocurra una inundación en el municipio de Campoalegre es mayor.

- La precipitación del entorno del rio Frio depende de la tensión del vapor.

\section{B. Reglas}

1 Tensión del vapor (alta) $\rightarrow$ precipitación (baja).

2 Nubosidad (alta) $\rightarrow$ tensión de vapor (baja).

3 Evaporación (media), temperatura (baja), caudal (alto) $\rightarrow$ precipitación (alta).

4 Humedad relativa (alta) $\rightarrow$ tensión de vapor (baja).

5 Nubosidad (alta), precipitación (alta) $\rightarrow$ tensión de vapor (baja) [12].

\section{CONCLUSIONES}

En el municipio de Campoalegre, la amenaza por inundación se presenta en los sectores contiguos al rio Magdalena, la quebrada La Sardinata, el rio Neiva, la quebrada La Ciénaga, la quebrada Otas, la quebrada Las Tapias, la quebrada Caraguaja, la quebrada San Isidro y la quebrada Rio Frio. Se evidencia este tipo de fenómenos de inundación cuando hay fuertes precipitaciones, estas hacen que aumente el caudal de las fuentes que discurren de la zona montañosa y en la parte de menor pendiente en la zona de piedemonte, en estas zonas tienden a desbordarse inundando las áreas aledañas.

En los últimos años se están presentando varios impactos en el municipio de Campoalegre, asociados al cambio climático, como lo son: fuertes incrementos de la temperatura, así como una tendencia a la disminución de la precipitación acumulada anual y de precipitaciones de alta intensidad (como los aguaceros); estos últimosue provocaron la inundación del 22 de febrero del 2017.

Analizando los datos suministrados del IDEAM en el software experto en minería de datos (Weka), se observó que la variable que está ligada a la inundación es la precipitación, debido a que, si es alta, el grado de inundación es mayor; el resto de variables afectarían directamente la precipitación.

\section{AGRADECIMIENTOS}

A la profesora Jasmidt Vera Cuenca, docente de la Universidad Surcolombiana, por su apoyo en la elaboración de este artículo. Al profesor Oswaldo Delgado, también docente de la Universidad Surcolombiana, por su apoyo y acompañamiento en la utilización de los aplicativos y el análisis de los resultados.

\section{REFERENCIAS}

[1] Ball State Undergraduate Mathematics Exchange, vol. 10, no. 1, 2016. [p. 1-15] [En línea]. Disponible en: https://lib.bsu.edu/beneficen cepress/mathexchange/10-01/index.html

[2] N. Sánchez Santillán, y R. Garduño López, “El clima, la ecología y el caos desde la perspectiva de la teoría general de los sistemas", Ingeniería. Investigación y Tecnología, vol. 8, no. 3, pp. 183195, 2007. [En línea]. Disponible en: https:// www. redalyc.org/pdf/404/40480305.pdf

[3] A. Nava, y D. Cortés, “Hidráulica de Ríos. Morfología, técnicas de medición y muestreo en ríos", Trabajo de grado (Pregrado), Instituto Politécnico Nacional, Escuela Superior de Ingeniería y Arquitectura, Naucalpan de Juárez, México, 2009. [En línea]. Disponible en: http://tesis.ipn.mx:8080/xmlui/handle/ $123456789 / 4632$

[4] M. C. Gallastegui, "El cambio climático y la importancia de las matemáticas", Matematicalia, vol. 6, no. 2, 2010. [En línea]. Disponible en: https://dialnet.unirioja.es/servlet/ articulo?codigo $=3238405$

[5] A. Triviño Pérez, y S. Ortiz Rojas, "Metodología para la modelación distribuida de la escorrentía superficial y la delimitación de zonas inundables en ramblas y ríos-rambla mediterráneos", Investigaciones geográficas, no. 35, pp. 67-83, 2004. doi: 10.14198/INGEO2004.35.08

[6] Alcaldía de Campoalegre, Plan básico de ordenamiento territorial, [En línea]. Disponible en: https://www.campoalegre-huila.gov.co/ Transparencia/Plan\%20basico\%200rdenamiento/PBOT / 03\%20DIAGNOSTICO\%20PBOT\%20 CAMPOALEGRE.pdf 
[7] Instituto de Hidrología, Meteorología y Estudios Ambientales, Metodología de la operación estadística variables meteorológicas [16-may-2018], Bogotá: IDEAM. Colombia. [En línea]. Disponible en: http://www.ide am.gov.co/documents/11769/72085840/ Documento+metodologico+variables+meteorologicas.pdf / 8a71a9b4-7dd7-4af4-b98e9b1eda3b8744

[8] Agencia Estatal de Meteorología, Manual de usos de términos meteorológicos, Madrid: AEMET, 2014. [En línea]. Disponible en: https:// www.aemet.es/documentos/es/eltiempo/ prediccion/comun/Manual_de_uso_de_terminos_met_2015.pdf

[9] H. E. Sarochar, Introducción a la meteorología general, Buenos Aires: Universidad Nacional de La Plata. [En línea, 20-nov-2018]. Disponible: http://extension.fcaglp.unlp.edu.ar/ content/obs/descargas/Introduccion_a_la_ Meteorologia_l.pdf

[10] A. K. López Sánchez, "Revaloración de las características de la arquitectura vernácula para su incorporación en la arquitectura contemporánea de la región de Juchitán", Tesis de grado (Maestría), Universidad Autónoma Metropolitana, México D.F., 2013. [En línea]. Disponible en: https: //core.ac.uk/download/pdf/128738892.pdf

[11] Master Oficial en Clima, Energía y Riesgo Ambiental, Clase práctica de Dinámica atmosférica: Ejecución del Modelo Regional Climático REMO, curso 2010-2011. [En línea]. Disponible en: http://www3.uah.es/clima/staff/gianni/doc/ practicas/practica1_ejecucion_modelo.pdf

[12] L. Timbe, y P. Willems, "Desempeño de modelos hidráulicos $1 \mathrm{D}$ y $2 \mathrm{D}$ para la simulación de inundaciones", Maskana, vol. 2, no. 1, pp. 91-98, 2011. doi: $10.18537 / \mathrm{mskn} .02 .01 .06$

[13] D. García, Manual de Weka, s.f. [En línea]. Disponible en: https: / / knowledgesociety.usal. es/sites/default/files/MANUAL\%20WEKA.pdf

[14] L. Aristizábal, y B. Oviedo, Guía de procedimiento para la generación de escenarios de cambio climático regional y local a partir de los modelos globales, Bogotá: IDEAM, 2010.

[15] Cristancho,A. andNavarro, D., 2014. DIRECCIONAMIENTO ESTRATÉGICO DE LA ACTIVIDAD LADRILLERA EN EL MUNICIPIO DE CAMPOALEGRE (HUILA) 2014-2017. [En línea]. Disponible en: https: / / bibliotecadigital.univalle.edu.co/ bitstream/handle/10893/10006/CB-0520909. pdf?sequence $=1$

[16] Humedad en la afmósfera (s.f). [En línea]. Disponible en: http://www.uaaan.mx/ jaflo$\mathrm{res} /$ humedad/humedad.pdf [20 abril 2021].

[17] Precipitación. Capítulo 2. [En línea]. Disponible en: http://www.biblioteca.udep.edu.pe/ bibvirudep/tesis/pdf/1_136_147_89_1257.pdf [20 abril 2021]

[18] Temperatura. Capítulo 4 (s.f). [En línea]. Disponible en: http://nimbus.com.uy/weather/ Cursos/Curso_2006/Textos\%20complementarios/Meteorologia\%20descriptiva_Inzunza/ cap4_Inzunza_Temperatura.pdf [20 abril 2021]

[19] A. Tejada Martínez, I. Méndez, N. Rodríguez, E. Tejada-Zacarías. "LA HUMEDAD EN LA ATMÓSFERA. Bases físicas, instrumentos y aplicaciones". Universidad de Colima, México 2018. [En línea]. Disponible en: http://ww.ucol. $\mathrm{mx} /$ content/publicacionesenlinea/adjuntos/ La-humedad-en-la-atmosfera_466.pdf 